\title{
Diacronie
}

Studi di Storia Contemporanea

$\mathrm{N}^{\circ} 29,1$ | 2017

"Crash test"

\section{Nota introduttiva n. 29 - marzo 2017}

Jacopo Bassi e Fausto Pietrancosta

\section{OpenEdition}

\section{Journals}

\section{Edizione digitale}

URL: http://journals.openedition.org/diacronie/4878

DOI: 10.4000/diacronie.4878

ISSN: 2038-0925

\section{Editore}

Association culturelle Diacronie

\section{Notizia bibliografica digitale}

Jacopo Bassi e Fausto Pietrancosta, « Nota introduttiva n. 29 - marzo 2017 », Diacronie [Online], № 29, 1 | 2017, Messo online il 29 mars 2017, consultato il 24 septembre 2020. URL : http:// journals.openedition.org/diacronie/4878; DOI : https://doi.org/10.4000/diacronie.4878

Questo documento è stato generato automaticamente il 24 settembre 2020.

Creative Commons License 
Nota introduttiva n. 29 - marzo 2017

Jacopo Bassi e Fausto Pietrancosta

\section{"Crash test"}

Continuità, discontinuità, persistenze e rotture nelle dinamiche della storia contemporanea 
1 Il numero 29 di Diacronie. Studi di Storia contemporanea intende proporre una discussione a più livelli sui concetti di continuità e recupero dei legami con le vicende, le visioni, i tracciati impressi dalle persone al corso delle storia, in una prospettiva comparativa con le improvvise dinamiche che sopraggiungono a rompere il quotidiano

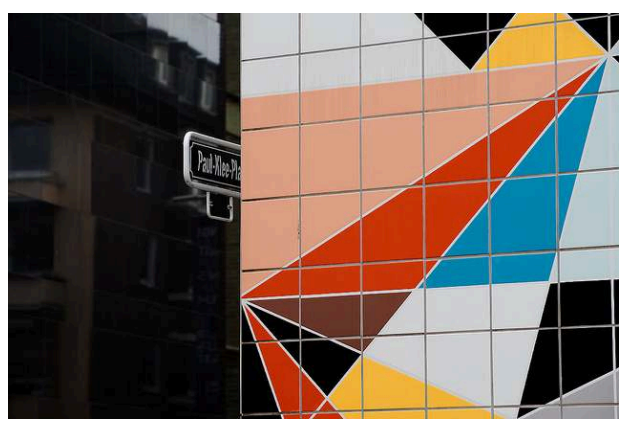
fluire della vita politica, economica e culturale delle società contemporanee tra XIX e XX secolo. Le eredità di lungo corso che la storia ci ha lasciato si confrontano in quest'ottica con le crisi e le rotture congiunturali degli assetti consolidati nel tempo, a volte in modo imprevisto, virulento e impetuoso, altre in modo graduale e per certi versi impercettibile, ma in ogni caso in modo tale da imprimere una svolta nelle strutture fondamentali dei vari ambiti della società, modificandola spesso radicalmente.

José D'Assunção Barros nel suo articolo prende in analisi l'egualitarismo nella letteratura utopica del XIX secolo, soffermandosi in particolare sulla società immaginaria ideata da Étienne Cabet, nel romanzo intitolato Voyage en Icarie (1842). La sua analisi è preceduta da una breve introduzione alla letteratura utopica a partire dal XVI secolo tesa al recupero, in chiave comparativa, delle proposte di autori quali Moro, Campanella, Francis Bacon, Fourier, Saint-Simon e Robert Owen. La riflessione proposta sui modelli di distribuzione dell'uguaglianza, si sviluppa in tal senso prendendo in considerazione le riflessioni di Norberto Bobbio e Amartya Sen su questo tema. La contestualizzazione dell'opera di Cabet si inquadra nel tentativo di gettare un ponte tra istanze letterarie e sociali, testimonianza di un legame forte tra temi culturali e società, e del grande interesse tra i lettori del tempo per questi temi che fornirono un contributo ideale fondamentale per la realizzazione di specifiche esperienze utopiche.

Giorgio Sacchetti nel suo Guerra e miniera prende in esame l'impatto che la Toscana delle industrie estrattive riuscì ad imprimere nel corso delle due guerre mondiali ritagliandosi, attraverso un contributo rilevante all'approvvigionamento del sistema produttivo italiano, un ruolo socio-economico di livello nazionale, che ebbe un'importanza cruciale soprattutto per quanto riguarda il settore energetico. Partendo da questo significativo caso-studio e analizzando alcune vertenze, il saggio si concentra così sul tema del lavoro in miniera, dei suoi forti legami col tessuto sociale e col territorio, pur in un momento di crisi e di violenta rottura rappresentato dalle emergenze belliche del 1915-18 e del 1939-45. L'analisi svolta si sofferma soprattutto sull'organizzazione produttiva, sulle modalità del reclutamento e sulla disciplina nelle differenti mobilitazioni industriali, sui contratti stipulati, sul trattamento salariale, sulle condizioni di lavoro e la salubrità, senza trascurare i temi dell'occupazione tedesca e della gestione partigiana dei siti minerari.

4 L'argomento affrontato da Francesco Landolfi nel suo articolo ci introduce ad uno dei momenti più traumatici della storia politica italiana: il sequestro dell'onorevole Aldo Moro da parte delle Brigate rosse. In tal senso questo evento e la sua drammatica conclusione hanno rappresentato un reale momento di crisi ed elemento di rottura delle dinamiche politiche e istituzionali, in grado di avviare una nuova fase della vita del paese. La lotta portata avanti dalle istituzioni contro il terrorismo brigatista 
trascinò infatti l'intera nazione in una situazione di latente guerra civile, fino a determinare un diverso corso per la politica nazionale e ad avviare una nuova fase per la storia italiana degli ultimi decenni. Nello specifico emerge la figura criminale di Antonio Chichiarelli e viene analizzato il caso del falso comunicato n. 7, attraverso il quale emergono i possibili legami operativi che potrebbero essersi instaurati tra malavita, Brigate rosse e agenzie di sicurezza all'interno di una vicenda ancora oggi non del tutto risolta.

5 Il periodo caratterizzato dall'esilio e dal ritorno di Perón al potere in Argentina (1955-1973), analizzato nell'articolo di Monica Bartolucci attraverso i movimenti studenteschi, permette di approfondire la nostra conoscenza su di un gruppo di personaggi che, dopo un breve protagonismo in politica, fu vittima della repressione statale voluta dalla dittatura militare del 1976. Durante questo periodo, il Departamento de Inteligencia de la Policía de la Provincia de Buenos Aires (DIPBA), la cui sede era a La Plata, riceveva quotidianamente informazioni dettagliate sull'attività degli agenti locali. Questi ultimi si infiltravano nelle riunione studentesche, di carattere politico o sindacale, ma anche in semplici manifestazioni, comizi o conferenze, con l'obiettivo di catalogare i singoli partecipanti in base alla propria "pericolosità". L'analisi di questa silenziosa sorveglianza ci permetterà di gettare luce su uno degli aspetti più traumatici della storia di questo paese, ed essenziale per comprendere il sistema di repressione portata avanti dal regime militare argentino negli anni Settanta.

6 Anche l'articolo di Pablo Baisotti si occupa della storia di questo paese. La guerra delle isole Falkland a sua volta rappresentò un trauma capace di comportare una rottura delle relazioni diplomatiche tra l'Argentina e la Gran Bretagna, e dunque nei legami di lungo corso che avevano caratterizzato i rapporti tra questi due paesi. I tentativi di ricomposizione delle relazioni e i relativi progressi che ne conseguirono prima e dopo il conflitto furono comunque esigui, soprattutto se rapportati al principale obiettivo dell'Argentina e cioè la possibilità di discutere in merito alla sovranità sulle isole. Questa, a tal scopo, promosse una diplomazia "informale" che si sviluppò di conseguenza e favorì quella opportuna "flessibilità", accompagnata da adeguate dimostrazioni di buona volontà, sulla quale si basarono i colloqui nei numerosi incontri, nei quali entrambi i paesi cercarono di arrivare ad una soluzione di compromesso con l'obiettivo di conciliare le proprie posizioni.

\section{Tavola rotonda. Wikipedia e le scienze storiche}

7 Diacronie ritorna su uno dei temi già affrontati in diverse occasioni come, ad esempio, nel numero 10: il rapporto fra storia e web. Wikipedia in questo caso è oggetto di un dibattito a sei voci - con profili differenti e rappresentative di altrettanti punti di vista - in forma di "Tavola rotonda". Ad una prima riflessione di Tommaso Baldo (in gran parte basata sulla riflessione scaturita dal collettivo Nicoletta Bourbaki) fanno seguito i commenti di cinque autori, conchiusi da un commento finale di Baldo. L'intento di questa prima "tavola rotonda" è riflettere non solamente, e non tanto, sui limiti e sulle potenzialità dell'"enciclopedia libera", quanto sull'interpretazione che va data di un progetto che è strutturalmente in fieri. La stessa definizione di Wikipedia (progetto collettivo/enciclopedia/social media/...) impiegata, implica una presa di posizione e un'interpretazione dell'oggetto in questione. Quale rapporto si viene a creare, dunque, tra Wikipedia e le scienze storiche? Quale interazione è possibile in un contesto che è diventato arena di scontro fra visioni differenti e modi diversi di "fare storia"? Si può 
pensare a una modalità di narrazione storica differente da quelle tradizionali o l'“enciclopedia libera" è destinata ad essere forgiata da dinamiche legate all'affermazione del "punto di vista neutrale"?

$\mathrm{Su}$ questi ed altri interrogativi si soffermano i partecipanti alla tavola rotonda "Wikipedia e le scienze storiche" che vi proponiamo.

\section{PICO. Il corporativismo}

9 Gli articoli del Progetto Interculturale COoperativo (Pico) di questo numero affrontano il tema del corporativismo. La rassegna di Diacronie sulla storiografia internazionale tradotta in lingua italiana per questo numero 29 fa ricorso alla produzione di due dei membri del neonato comitato di direzione della rivista: Spyridon Ploumidis e João Fábio Bertonha. L'articolo di Ploumidis analizza le specificità del corporativismo ellenico, contestualizzandolo nel clima sociale ed economico del periodo compreso tra le due guerre. L'adozione di misure corporativiste va perciò ricondotta al clima di generale sfiducia nei confronti del liberalismo che andava diffondendosi negli anni Venti e Trenta in Europa. João Fábio Bertonha prende invece in esame la figura di Miguel Reale, tra i maggiori esponenti dell'integralismo brasiliano, che aderì successivamente al progetto di Getúlio Vargas. La concezione corporativista dello Stato di Reale - come dimostra l'autore - era ispirata dal fascismo della prima ora, di cui il giurista brasiliano era un profondo conoscitore. Anche per questa ragione il vincolo che lo legò al fascismo italiano si sciolse solamente nelle fasi finali del regime italiano.

Chiudono il numero le consuete recensioni.

Un ringraziamento particolare a Stavros Kouroufexis per il preciso e approfondito lavoro di traduzione dell'articolo di Spyridon Ploumidis e a Luca Giuseppe Manenti per la paziente e attenta rilettura della traduzione dell'articolo di José D'Assunção Barros. Buona lettura,

13 Jacopo Bassi, Fausto Pietrancosta

\section{AUTORI}

\section{JACOPO BASSI}

Jacopo Bassi ha conseguito la Laurea Triennale in «Storia del mondo contemporaneo» presso l'Università di Bologna sostenendo una tesi in Storia e istituzioni della Chiesa ortodossa dal titolo Tra Costantinopoli e Atene: Il passaggio delle diocesi dell'Epiro all'amministrazione della Chiesa di Grecia e la 'Praxis' del 1928; presso lo stesso ateneo, nel 2008, ha discusso la tesi specialistica in Storia della Chiesa dal titolo Epiro crocifisso o liberato? La Chiesa ortodossa in Epiro e in Albania meridionale nel XX secolo (1912-1967). Attualmente collabora con le case editrici Il Mulino e Zanichelli. URL: < http://www.studistorici.com/2009/02/24/jacopo_bassi/ > 


\section{FAUSTO PIETRANCOSTA}

Fausto Pietrancosta ha conseguito il titolo di dottore di ricerca in Storia presso l'Università di Bologna con una tesi inerente le relazioni tra istituzioni politiche e intervento pubblico in economia nella prospettiva del coordinamento tra amministrazioni centrali ed ente regionale siciliano. Già dottore magistrale in Storia d'Europa, presso la stessa Università con una tesi in Storia dello Stato italiano sul coordinamento costituzionale e l'avvio dell'autonomia regionale siciliana, ha svolto attività di ricerca presso l'Archivio Storico e la biblioteca dell'Istituto Luigi Sturzo di Roma, presso l'Assemblea regionale siciliana, proseguendo poi l'attività di ricerca presso gli archivi degli enti pubblici economici in Sicilia e presso la biblioteca SVIMEZ di Roma. I suoi interessi sono rivolti allo studio dell'evoluzione storica delle autonomie regionali nell'Italia del secondo dopoguerra e delle politiche di intervento a favore dello sviluppo industriale nel Mezzogiorno.

URL: < http://www.studistorici.com/2008/09/14/fausto-pietrancosta/ > 